\title{
ATTENTION-BASED SCALING ADAPTATION FOR TARGET SPEECH EXTRACTION
}

\author{
Jiangyu Han ${ }^{1,2}$, Wei Rao ${ }^{2}$, Yanhua Long ${ }^{1}$, Jiaen Liang ${ }^{3}$ \\ ${ }^{1}$ Shanghai Normal University, Shanghai, China \\ ${ }^{2}$ Tencent Ethereal Audio Lab, Tencent Corporation, Shenzhen, China \\ ${ }^{3}$ Unisound AI Technology Co., Ltd., Beijing, China
}

\begin{abstract}
The target speech extraction has attracted widespread attention in recent years. In this work, we focus on investigating the dynamic interaction between different mixtures and the target speaker to exploit the discriminative target speaker clues. We propose a special attention mechanism without introducing any additional parameters in a scaling adaptation layer to better adapt the network towards extracting the target speech. Furthermore, by introducing a mixture embedding matrix pooling method, our proposed attention-based scaling adaptation (ASA) can exploit the target speaker clues in a more efficient way. Experimental results on the spatialized reverberant WSJ0 2-mix dataset demonstrate that the proposed method can improve the performance of the target speech extraction effectively. Furthermore, we find that under the same network configurations, the ASA in a single-channel condition can achieve competitive performance gains as that achieved from two-channel mixtures with inter-microphone phase difference (IPD) features.
\end{abstract}

Index Terms - Target speech extraction, time-domain, attention, adaptation

\section{INTRODUCTION}

Speech separation aims to separate each source signal from the mixed speech. Many works have been proposed to perform the separation either in the time-frequency domain or the purely timedomain, such as deep clustering [1], deep attractor network [2], permutation invariant training (PIT) [3] and the recent convolutional time-domain audio separation network (Conv-TasNet) [4], etc. Our work aims to generalize the time-domain Conv-TasNet to the target speech extraction (TSE) tasks, because of its higher performances over most time-frequency domain methods [5-10]. Compared with the pure speech separation techniques, most TSE approaches require additional target speaker clues to guide the separation network to extract the speech of that speaker.

Many works have been proposed for the TSE target speaker adaptation, such as the SpeakerBeam-FE [11], SpEx [12], and timedomain SpeakerBeam (TD-SpeakerBeam) [13] employed a factorized adaptation layer, a concatenating adaptation layer, and a scaling adaptation layer to adjust the internal behavior of the separation network respectively. Although these adaptation methods showed promising results, their exploitations of the target speaker clues are somehow monotonous. Specifically, they average the speaker vectors over an adaptation or enrollment utterance to get a vector that

Work was done when Jiangyu Han was an intern at Tencent Ethereal Audio Lab. Yanhua Long is the corresponding author. The work is supported by the National Natural Science Foundation of China (Grant No.62071302 and No.61701306). provides a global bias for the target speaker, then, repeat the same vector for each frame adaptation of the mixed speech to drive the network towards extracting the target speech, no matter how the acoustic characteristics of the frame are related to the target speaker identity. Therefore, we think that a more reasonable target speaker bias should be able to adjust itself dynamically according to the acoustic interaction between the mixture and the target speaker.

In [14], an attention mechanism between the adaptation utterances and the mixed speech was proposed to dynamically compute a frame-wise speaker bias which is a weighted sum of target speaker embeddings. However, it was designed for the time-frequency domain TSE tasks, it is tricky to generalize such attention to the timedomain TSE tasks. Because the frames of the encoded representation for a waveform in the time-domain (e.g. 3199) is usually much larger than that in the time-frequency domain (e.g. 251). Such attention performed on these large frames may result in a very sparse distribution of their softmax probabilities, in which only several frames have big probabilities, while others are close to zero. In this case, the weighted sum of target speaker vectors can no longer provide a sufficient discriminative bias for extracting the target speech. Although the authors in [15] applied the same attention mechanism to the time-domain tasks, they only presented the results of the pretrained speaker embedder.

In this study, we design a novel attention mechanism to exploit the interaction between the adaptation utterance and different mixture speech for the time-domain TSE tasks. Different from [14], our attention is performed on the global bias vector of the target speaker and the embedding matrix of the mixed speech, because we want to adjust the target speaker bias dynamically according to the acoustic interaction between the mixture and target speaker at different time intervals. Rather than learning the context-dependent information in [14], we pay more attention to exploit the dynamic target speaker identity clues between different mixtures and the target speaker adaptation utterance. Furthermore, as our attention is directly performed between a vector and a matrix, unlike the traditional self-attention [16] with linear projection weights, there are no additional network parameters is introduced in our ASA, and it has a less computational cost and lower memory requirements than the attention performed on two matrices. All experiments are performed on the publicly available spatialized reverberant WSJ0 2-mix dataset. Results show that the proposed method improves the target speech extraction performance effectively, and the single-channel performances are comparable to that in multi-channel condition with IPD features [17].

The rest of this paper is organized as follows. In Section 2 we introduce arcthitecture of our baseline. In Section 3, we describe the principle of our proposed ASA. The experiments and results are presented in Section 4 and conclude in Section 5 


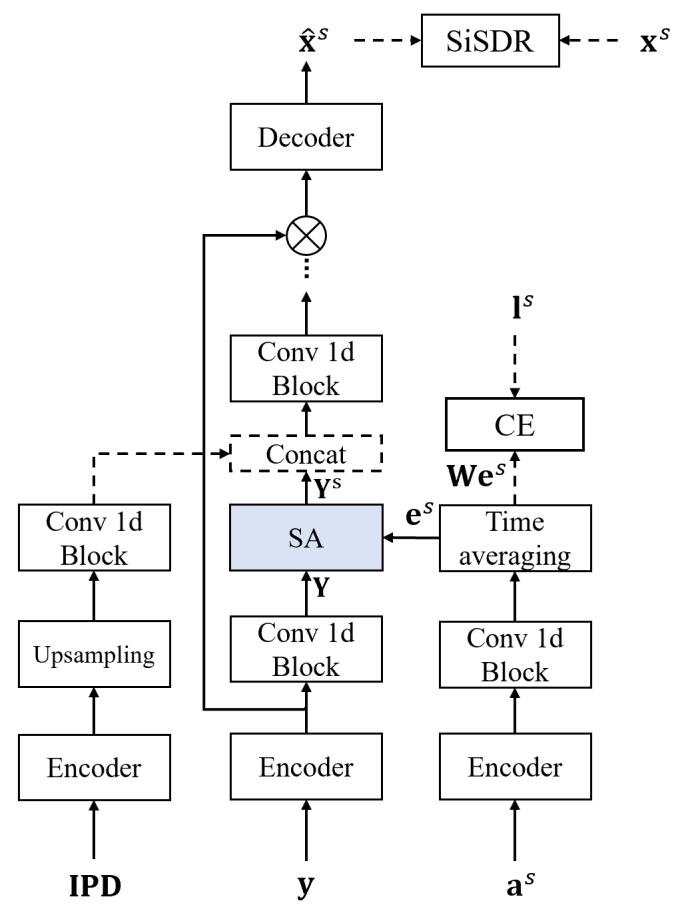

Fig. 1. The block diagram of the TD-SpeakerBeam with IPD. "SA" represents scalling adaptation.

\section{TIME-DOMAIN SPEAKERBEAM}

TD-SpeakerBeam is a very effective target speech extraction approach that has been recently proposed in [13]. The structure of TD-SpeakerBeam with IPD concatenation block is shown in Fig 1 It contains a $1 \mathrm{~d}$ convolution encoder, several convolution blocks, and a $1 \mathrm{~d}$ deconvolution decoder. The $\mathbf{y}, \hat{\mathbf{x}}^{s}$ and $\mathbf{a}^{s}$ are the mixture waveform, the extracted target speech waveform, and the adaptation utterance of the target speaker respectively.

The whole TD-SpeakerBeam network follows a similar configuration as Conv-TasNet [4] except for inserting a scaling adaptation (SA) layer [18] between the first and second convolution blocks to drive the network towards extracting the target speech. The output of the SA layer $\mathbf{Y}^{\mathbf{s}} \in \mathbb{R}^{N \times T}$ is obtained by simply performing an element-wise multiplication between the repeated target speaker embedding vectors $\left[\mathbf{e}^{s}, \mathbf{e}^{s}, \ldots, \mathbf{e}^{s}\right]_{N \times T}$ and the input $\mathbf{Y}$ as follows,

$$
\mathbf{Y}^{\mathrm{s}}=\left[\mathbf{e}^{s}, \mathbf{e}^{s}, \ldots, \mathbf{e}^{s}\right]_{N \times T} \odot \mathbf{Y}
$$

where the $\mathbf{e}^{s} \in \mathbb{R}^{N \times 1}$ is computed from a time-domain convolutional auxiliary network as shown in the right part of Fig 1 . and the $N$ is the dimension of the embedding vectors, $T$ is the number of frames of the convolutional output. Furthermore, as shown in the left part of Fig 1 TD-SpeakerBeam can also be extended to the multichannel TSE task by combining the IPD features (processed with a 1d convolutional layer, upsampling, and a convolution block) after the SA layer.

The whole network of TD-SpeakerBeam is trained jointly in an end-to-end multi-task way. The multi-task loss combines the scaleinvariant signal-to-distortion ratio (SiSDR) [19] as the signal reconstruction loss and cross-entropy as the speaker identification loss. The overall loss function is defined as,

$$
L\left(\Theta \mid \mathbf{y}, \mathbf{a}^{s}, \mathbf{x}^{s}, \mathbf{l}^{s}\right)=-\operatorname{SiSDR}\left(\mathbf{x}^{s}, \hat{\mathbf{x}}^{s}\right)+\alpha \operatorname{CE}\left(\mathbf{l}^{s}, \sigma\left(\mathbf{W e} \mathbf{e}^{s}\right)\right)
$$

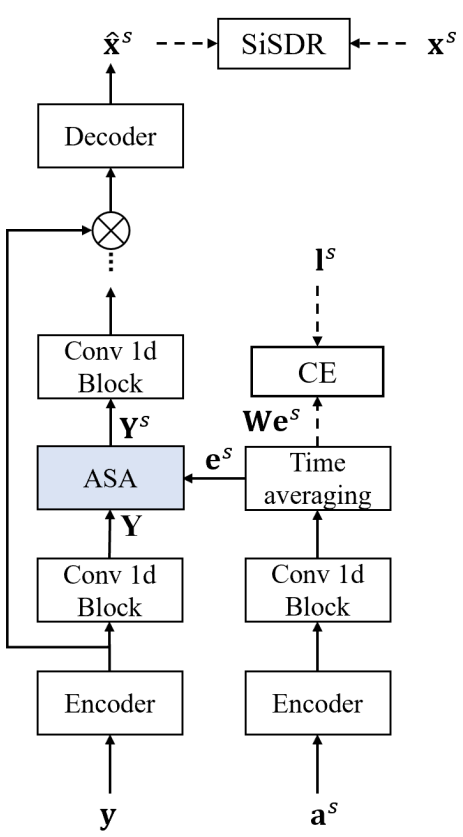

(a)

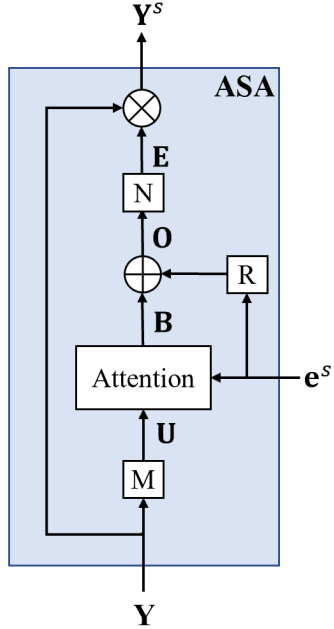

(b)
Fig. 2. The block diagram of (a) proposed method based on TDSpeakerBeam and (b) the structure of ASA. "M" represents mean pooling, " $\mathrm{R}$ " represents repeating, and "N" represents nearest upsampling.

where $\Theta$ represents the model parameters, $\mathbf{x}^{s}$ is the target speech, $\mathbf{l}^{s}$ is a one-hot vector representing the target speaker identity, $\alpha$ is a scaling parameter, $\mathbf{W}$ is a weight matrix and $\sigma(\cdot)$ is softmax operation. More details can be found in [13].

\section{PROPOSED METHOD}

\subsection{Overview}

The whole structure of the proposed method is shown in Fig 2 (a). All network architectures are the same as Fig 1 except for replacing the SA layer with our proposed attention-based scaling adaptation (ASA). As shown in Fig 2 (b), the ASA layer accepts the mixture embedding matrix $\mathbf{Y} \in \mathbb{R}^{N \times T}$ and the target speaker embedding vector $\mathbf{e}^{s}$ as inputs, and outputs the scaled mixture embedding matrix $\mathbf{Y}^{s} \in \mathbb{R}^{N \times T}$ whose each value is weighted by the dynamic target speaker bias $\mathbf{E}$. $N$ is the output dimension of the convolutional network, and $T$ is the number of frames of the convolutional output.

As our operations are performed in the time-domain, in order to get a high time-domain resolution, the convolutional kernel used to encode the waveform usually has a small size, which means the number frames of the convolution output $T$ has a large size. For example, assuming the input waveform has 32000 samples, the $1 \mathrm{~d}$ convolutional kernel size is 20 , stride is 10 and padding is 0 . Then the number of frames of the convolutional output will be 3199 . When performing the softmax over these frames, the obtained probability vector usually has a very sparse distribution. Such a sparse distribution will prevent the conventional attention mechanism from being used to effectively exploit the relationship between the mixture and the target speaker for the time-domain tasks. 


\subsection{Attention-based scaling adaptation}

Considering that the speaker characteristics of several consecutive frames are almost unchanged, we treat each non-overlapped consecutive $M$ frames as an integral part, by averaging each $M$ column vectors of the mixture embedding matrix $\mathbf{Y}$ to generate a speakerdependent vector $\mathbf{u}_{t}$. After such a mean pooling operation, we can obtain a speaker-dependent matrix $\mathbf{U}$, i.e.,

$$
\begin{aligned}
& \mathbf{u}_{t}=\frac{1}{M} \sum_{j=1}^{M} \mathbf{y}_{j+(t-1) M} \\
& \mathbf{U}=\left[\mathbf{u}_{1}, \mathbf{u}_{2}, \ldots, \mathbf{u}_{T_{m}}\right]
\end{aligned}
$$

where $\mathbf{U} \in \mathbb{R}^{N \times T_{m}}, T_{m}$ equals to $T / M, \mathbf{u}_{t} \in \mathbb{R}^{N \times 1}$ is the $t$-th column vector of $\mathbf{U}$, and $\mathbf{y}_{j} \in \mathbb{R}^{N \times 1}$ is the $j$-th column vector of $\mathbf{Y}$. By doing so, the new mixture embedding matrix $\mathbf{U}$ not only has a much smaller size than $\mathbf{Y}$, but also has a relatively compact acoustic representation to reflect the speaker dependent information, each $\mathbf{u}_{i}$ summaries the speaker information over several frames and represents a different dynamic bias to the target speaker.

Next, as shown in Fig2 (b), we take $\mathbf{U}$ and the target speaker embedding vector $\mathbf{e}^{s}$ as inputs of the attention module. The specific procedure is as follows:

$$
\begin{aligned}
\mathbf{d} & =\left(\mathbf{e}^{s}\right)^{\mathbf{T}} * \mathbf{U} \\
w_{t} & =\frac{e^{d_{t}}}{\sum_{i=1}^{T_{m}} e^{d_{i}}} \\
\mathbf{B} & =\mathbf{e}^{s} * \mathbf{w}
\end{aligned}
$$

where $*$ is the operation of matrix multiplication operation, $\mathbf{T}$ is the operation of transpose, $\mathbf{d} \in \mathbb{R}^{1 \times T_{m}}$ is a similarity vector that measures the correlation between $\mathbf{e}^{s}$ and $\mathbf{u}_{t}, d_{t}$ is the $t$-th element of $\mathbf{d}, w_{t}$ is the softmax of $d_{t}$ over $t \in\left[1, T_{m}\right], \mathbf{w} \in \mathbb{R}^{1 \times T_{m}}$ is the corresponding softmax vector, and $\mathbf{B} \in \mathbb{R}^{N \times T_{m}}$ is the attentionbased target speaker embedding matrix.

Each vector at frame $t$ in $\mathbf{B}$ can be regarded as a target speakerdependent dynamic bias $\mathbf{b}_{t}$, because in which the $\mathbf{e}^{s}$ is weighted by the similarity $w_{t}$ that exploits the dynamic target speaker interaction between the mixture and the adaptation utterance. In addition, as the dynamic bias $\mathbf{b}_{t}$ will be very small values if the mixture speech is occupied by the interferer, this bias may fail to be a good target speaker guider to supervise the whole network training.

In order to dynamically adjust the target speaker bias with discriminative speaker information, as shown in Fig 2(b), we repeat the target speaker embedding vector $\mathbf{e}^{s}$ and add it to the dynamic bias $\mathbf{b}_{t}$ to get the output $\mathbf{O}$, i.e.,

$$
\begin{aligned}
\mathbf{o}_{t} & =\mathbf{b}_{t}+\mathbf{e}^{s} \\
\mathbf{O} & =\left[\mathbf{o}_{1}, \mathbf{o}_{2}, \ldots, \mathbf{o}_{T_{m}}\right]
\end{aligned}
$$

where $\mathbf{O} \in \mathbb{R}^{N \times T_{m}}, \mathbf{o}_{t} \in \mathbb{R}^{N \times 1}$ is the $t$-th vector of $\mathbf{O}$ at frame $t$. This repeat operation in (7) assures the target speaker bias won't be too weak, while the attention mechanism provides a dynamic and more discriminative bias. Combining these two techniques, the target speaker embedding $\mathbf{O}$ can drive the network towards extracting the target speech more efficiently.

Next, we use the nearest upsampling algorithm to map the $\mathbf{O}$ to the same dimension as the original mixture embedding matrix $\mathbf{Y}$ to get the final target speaker embedding matrix $\mathbf{E} \in \mathbb{R}^{N \times T}$. Due to the nearest upsampling, the consecutive $M$ vectors (frames) of $\mathbf{E}$ have the same target speaker characteristics, which is consistent with our intuition, namely, the speaker characteristics won't change in a very short duration.

Finally, an element-wise multiplication between $\mathbf{Y}$ and $\mathbf{E}$ is used to adapt the network towards the target speaker, i.e.,

$$
\mathbf{Y}^{s}=\mathbf{Y} \odot \mathbf{E}
$$

where $\mathbf{Y}^{s} \in \mathbb{R}^{N \times T}$ is the output of the proposed attention-based scaling adaptation. It is worth noting that, unlike the traditional selfattention that needs additional learnable weights to project the key, query and value into high-level representation spaces, our ASA is very simple, and it is directly performed on the $\mathbf{U}$ and $\mathbf{e}^{s}$, no additional parameter is introduced.

\section{EXPERIMENTS AND RESULTS}

\subsection{Dataset}

Our experiments are performed on the simulated spatialized WSJ0 2-mix corpus [20]. All recordings are generated by convolving clean speech signals with room impulse responses simulated with the image method for reverberation time ranges from $0.2 \mathrm{~s}$ to $0.6 \mathrm{~s}$ [13]. We use the same way as in [21] to generate adaptation utterances of the target speaker. The adaptation utterance is selected randomly that to be different from the utterance in the mixture. The adaptation recordings used in our experiments are anechoic. The size of the training, validation, and test sets are $20 \mathrm{k}, 5 \mathrm{k}$, and $3 \mathrm{k}$ utterances, respectively. All of the data are resampled to $8 \mathrm{kHz}$ from the original $16 \mathrm{kHz}$ sampling rate.

\subsection{Configurations}

Our experiments are implemented based on the open source software [22]. We use the same hyper-parameters as the baseline TD-SpeakerBeam in [13]. We set $M=20$ in the ASA process. All experiments are performed using the SiSDR loss only $(\alpha=0$ in Eq.22) except when we mention the use of the multi-task loss (MTL), in which case we set $\alpha=0.5$ to balance the loss tradeoff between the SiSDR and cross-entropy. For those experiments with IPD combination, the IPD features are extracted using an STFT window of $32 \mathrm{msec}$ and hop size of $16 \mathrm{msec}$. All experiments are performed on the single-channel (first channel) recordings, except for the experiment with IPD features that extract from two-channel recordings. For the performance evaluation, both the signal-to-distortion ratio (SDR) of BSSeval [23] and the SiSDR [19] are used.

\subsection{Results and discussion}

\subsubsection{Baseline}

We take the single-channel TD-SpeakerBeam [13] (without IPD combination) as our baseline. Results are shown in Table 1 System (1) and (2) are the results given in [13]. As the source code of TD-SpeakerBeam is not open resource, we implemented it by ourselves and reproduced the results as shown in system (3) and (4) on the same WSJ0 2-mix corpus. We see that our reproduced results are slightly better than the ones in [13]. Moreover, by comparing (1) and (2), or (3) and (4), there is a big performance gap between the single-channel and two-channel TSE tasks, the IPD features extracted from two-channel recordings are effective to improve the TSE performance. In addition, we find that the TSE on the same gender conditions are much more challenge than the mix-gender condition. 
Table 1. SDR / SiSDR (dB) performance of TD-SpeakerBeam (TSB). "IPD" represents the system with internal combination of IPD spatial features, "F" represents female, "M" represents male, and "Avg" represents the average results. Bold-fonts indicate the best performance.

\begin{tabular}{l|c|c|c|c|c}
\hline System & IPD & FF & MM & FM & Avg \\
\hline (1) TSB [14] & - & $9.13 /-$ & $9.47 /-$ & $12.77 /-$ & $11.17 /-$ \\
(2) & $\checkmark$ & $\mathbf{1 0 . 1 7 / -}$ & $10.30 /-$ & $12.49 /-$ & $11.45 /-$ \\
\hline (3) TSB (our) & - & $9.43 / 8.84$ & $10.02 / 9.52$ & $12.54 / 12.06$ & $11.26 / 10.76$ \\
(4) & $\checkmark$ & $10.01 / 9.46$ & $\mathbf{1 0 . 5 1} / \mathbf{1 0 . 0 2}$ & $\mathbf{1 2 . 8 0} / \mathbf{1 2 . 3 1}$ & $\mathbf{1 1 . 6 5 / 1 1 . 1 5}$ \\
\hline
\end{tabular}

\subsubsection{Results of ASA with SiSDR loss}

Table 2 shows the experimental results of our proposed ASA with SiSDR loss as the system training criterion. System (1) is our baseline. In order to examine the effects of the proposed mean pooling operation that used in the ASA, we remove the blocks of mean pooling $(\mathrm{M})$ and nearest upsampling $(\mathrm{N})$ used in Fig 2 (b). The proposed attention mechanism is performed directly between the mixture embedding matrix $\mathbf{Y}$ and the target speaker embedding vector $\mathbf{e}^{s}$. The results are shown in system (2). Compared with the baseline (system (1)), it's clear to see that system (2) achieves better performance under all gender-mixing conditions. The results of system (2) indicate that the dynamic interaction between the mixture and the target speaker is helpful to produce a more discriminative speaker bias to guide the network to extract the target speech.

Table 2. SDR / SiSDR (dB) performance of the proposed attenion based scaling adaptation (ASA) method. "MP" represents the mean pooling in ASA.

\begin{tabular}{l|c|c|c|c}
\hline System & FF & MM & FM & Avg \\
\hline (1) TSB & $9.43 / 8.84$ & $10.02 / 9.52$ & $12.54 / 12.06$ & $11.26 / 10.76$ \\
\hline (2) ASA & $9.78 / 9.23$ & $10.36 / 9.86$ & $12.78 / 12.29$ & $11.55 / 11.05$ \\
(3) ASA (MP) & $9.83 / 9.26$ & $10.47 / 9.97$ & $12.89 / 12.40$ & $11.65 / 11.15$ \\
\hline
\end{tabular}

(4) Para [24] $|10.83 / 10.21| 11.52 / 10.99|13.12 / 12.61| 12.26 / 11.72$ (5) Para (ASA) $11.50 / 10.87|12.01 / 11.48| 13.56 / 13.06 \mid 12.75 / 12.22$

Moreover, as shown in the system (3), the TSE performance can be further improved by introducing the mean pooling operation mentioned in Section 3.2 Summarizing the speaker information prior to the attention calculation can enhance the ability of exploiting the dynamic target speaker-dependent information between different input mixtures and the given adaptation utterance of the target speaker. Therefore, the mean pooling is set to the default configuration for the rest of our experiments. Compared with the baseline (system (1)), the proposed ASA (system(3)) achieves $4.2 / 4.7 \%, 4.5 / 4.7 \%$ and $2.8 / 1.9 \%$ relative improvements in SDR / SiSDR for femalefemale, male-male, female-male conditions respectively. It indicates that the ASA can effectively enhance the target speech extraction, especially for the same-gender mixtures. Furthermore, by comparing the results of system (4) in Table 1 and system (3) in Table 2, the proposed system with ASA for single-channel TSE task achieves competitive performance as the TD-SpeakerBeam with IPD for the multi-channel task. In addition, please note that the ASA does not introduce any additional learnable parameters like linear transformation weight of query, key, and value in self-attention [16]. The proposed ASA only contains pure mathematical operations. These promising results indicate that our proposed ASA is effective to im-
Table 3. SDR / SiSDR (dB) performance of the proposed attention based scaling adaptation (ASA) method with multi-task loss (MTL).

\begin{tabular}{l|c|c|c|c}
\hline System & FF & MM & FM & Avg \\
\hline (1) TSB & 9.43 / 8.84 & $10.02 / 9.52$ & $12.54 / 12.06$ & $11.26 / 10.76$ \\
(2) TSB (MTL) & $9.66 / 9.09$ & 10.33 / 9.84 & $12.75 / 12.26$ & $11.51 / 11.00$ \\
\hline (3) ASA (MTL) & $\mathbf{9 . 9 3 ~ / ~ 9 . 3 5}$ & $\mathbf{1 0 . 6 3} / \mathbf{1 0 . 1 3}$ & $\mathbf{1 2 . 9 2}$ / 12.43 & $\mathbf{1 1 . 7 3} / \mathbf{1 1 . 2 2}$ \\
\hline
\end{tabular}

prove the discrimination of target speaker clues for TSE tasks.

Although as suggested in [14], the local dynamics and the temporal structure will be lost due to the averaging operation, the averaging can provide a more robust speaker bias vector than a single frame vector. Experimental results show that the local mean pooling and the nearest upsampling operations are beneficial for ASA to exploit the target speaker clues in a more efficient way. We also tried applying the attention mechanism from [14] to the TSB structure, but the training process had difficulties to converge and so we stopped it after several epochs.

Furthermore, we also investigate effects of ASA on two-channel parallel encoder based TSB system [24, 25] which directly sums the waveform encodings of each input channel to enhance the mixture representation. The adaptation methods of system (4) and (5) are SA and ASA with mean pooling. Results show that the extraction performance can be further improved by introducing the proposed ASA mechanism.

\subsubsection{Results of ASA with multi-task loss}

We also investigated the performance of ASA with multi-task loss. Results are shown in Table 3 System (1) is the TD-SpeakerBeam trained with SiSDR loss function, and system (2) and (3) are the TD-SpeakerBeam and the proposed ASA system (with mean pooling) trained with a multi-task loss respectively. Results show that even using a multi-task loss, the proposed ASA still achieves better performances than the baselines. Note that in this case, we scaled the attention-based target speaker embedding matrix $\mathbf{B}$ by $\sqrt{N}$ as we found this combination can result in better performance.

\section{CONCLUSION}

In this work, we propose a novel target speaker adaptation technique for time-domain target speech extraction tasks. A special attention mechanism is designed to effectively exploit the dynamic target speaker-dependent interaction between different mixtures and the given adaptation utterance. This dynamic information can improve the target speaker clues and its discriminations for target speech extraction. Experiments on the spatialized WSJ0 2-mix corpus demonstrate that our proposed method effectively improved the TD-SpeakerBeam for target speech extraction. Furthermore, it is surprising to find that the single-channel performance gains result from the proposed ASA are competitive with the multi-channel TDSpeakerBeam with IPD features. Our future work will focus on how to combine the proposed method with the multi-task loss in a more appropriate way.

\section{REFERENCES}

[1] J. R. Hershey, Z. Chen, J. Le Roux, and S. Watanabe, "Deep clustering: Discriminative embeddings for segmentation and 
separation," in 2016 IEEE International Conference on Acoustics, Speech and Signal Processing (ICASSP). IEEE, 2016, pp. 31-35.

[2] Z. Chen, Y. Luo, and N. Mesgarani, "Deep attractor network for single-microphone speaker separation," in 2017 IEEE International Conference on Acoustics, Speech and Signal Processing (ICASSP). IEEE, 2017, pp. 246-250.

[3] M. Kolbæk, D. Yu, Z. H. Tan, and J. Jensen, "Multitalker speech separation with utterance-level permutation invariant training of deep recurrent neural networks," IEEE/ACM Transactions on Audio, Speech, and Language Processing, vol. 25, no. 10, pp. 1901-1913, 2017.

[4] Y. Luo and N. Mesgarani, "Conv-TasNet: Surpassing ideal time-frequency magnitude masking for speech separation," IEEE/ACM transactions on audio, speech, and language processing, vol. 27, no. 8, pp. 1256-1266, 2019.

[5] D. Ditter and T. Gerkmann, "A multi-phase gammatone filterbank for speech separation via TasNet," in 2020 IEEE International Conference on Acoustics, Speech and Signal Processing (ICASSP). IEEE, 2020, pp. 36-40.

[6] J. Heitkaemper, D. Jakobeit, C. Boeddeker, L. Drude, and R. Haeb-Umbach, "Demystifying TasNet: A dissecting approach," in 2020 IEEE International Conference on Acoustics, Speech and Signal Processing (ICASSP). IEEE, 2020, pp. 6359-6363.

[7] T. Ochiai, M. Delcroix, R. Ikeshita, K. Kinoshita, T. Nakatani, and S. Araki, "Beam-TasNet: Time-domain audio separation network meets frequency-domain beamformer," in 2020 IEEE International Conference on Acoustics, Speech and Signal Processing (ICASSP). IEEE, 2020, pp. 6384-6388.

[8] M. Pariente, S. Cornell, A. Deleforge, and E. Vincent, "Filterbank design for end-to-end speech separation," in 2020 IEEE International Conference on Acoustics, Speech and Signal Processing (ICASSP). IEEE, 2020, pp. 6364-6368.

[9] R. Gu, S.-X. Zhang, L. Chen, Y. Xu, M. Yu, D. Su, Y. Zou, and D. Yu, "Enhancing end-to-end multi-channel speech separation via spatial feature learning," in 2020 IEEE International Conference on Acoustics, Speech and Signal Processing (ICASSP). IEEE, 2020, pp. 7319-7323.

[10] S. Sonning, C. Schüldt, H. Erdogan, and S. Wisdom, "Performance study of a convolutional time-domain audio separation network for real-time speech denoising," in 2020 IEEE International Conference on Acoustics, Speech and Signal Processing (ICASSP). IEEE, 2020, pp. 831-835.

[11] M. Delcroix, K. Zmolikova, K. Kinoshita, A. Ogawa, and T. Nakatani, "Single channel target speaker extraction and recognition with speaker beam," in 2018 IEEE International Conference on Acoustics, Speech and Signal Processing (ICASSP). IEEE, 2018, pp. 5554-5558.

[12] C. Xu, W. Rao, E. S. Chng, and H. Li, "SpEx: Multi-scale time domain speaker extraction network," IEEE/ACM Transactions on Audio, Speech, and Language Processing, vol. PP, no. 99, pp. 1-1, 2020.

[13] M. Delcroix, T. Ochiai, K. Zmolikova, K. Kinoshita, N. Tawara, T. Nakatani, and S. Araki, "Improving speaker discrimination of target speech extraction with time-domain speakerbeam," in 2020 IEEE International Conference on Acoustics, Speech and Signal Processing (ICASSP). IEEE, 2020, pp. 691-695.
[14] X. Xiao, Z. Chen, T. Yoshioka, H. Erdogan, C. Liu, D. Dimitriadis, J. Droppo, and Y. Gong, "Single-channel speech extraction using speaker inventory and attention network," in 2019 IEEE International Conference on Acoustics, Speech and Signal Processing (ICASSP). IEEE, 2019, pp. 86-90.

[15] X. Ji, M. Yu, C. Zhang, D. Su, T. Yu, X. Liu, and D. Yu, "Speaker-aware target speaker enhancement by jointly learning with speaker embedding extraction," in 2020 IEEE International Conference on Acoustics, Speech and Signal Processing (ICASSP). IEEE, 2020, pp. 7294-7298.

[16] A. Vaswani, N. Shazeer, N. Parmar, J. Uszkoreit, L. Jones, A. N. Gomez, L. Kaiser, and I. Polosukhin, "Attention is all you need," arXiv preprint arXiv:1706.03762, 2017.

[17] Z. Chen, X. Xiao, T. Yoshioka, H. Erdogan, J. Li, and Y. Gong, "Multi-channel overlapped speech recognition with location guided speech extraction network," in Spoken Language Technology Workshop (SLT). IEEE, 2018, pp. 558-565.

[18] M. Delcroix, K. Zmolikova, T. Ochiai, K. Kinoshita, S. Araki, and T. Nakatani, "Compact network for speakerbeam target speaker extraction," in 2019 IEEE International Conference on Acoustics, Speech and Signal Processing (ICASSP). IEEE, 2019, pp. 6965-6969.

[19] J. Le Roux, S. Wisdom, H. Erdogan, and J. R. Hershey, "SDRhalf-baked or well done?," in 2019 IEEE International Conference on Acoustics, Speech and Signal Processing (ICASSP). IEEE, 2019, pp. 626-630.

[20] Z.-Q. Wang, J. Le Roux, and J. R. Hershey, "Multi-channel deep clustering: Discriminative spectral and spatial embeddings for speaker-independent speech separation," in 2018 IEEE International Conference on Acoustics, Speech and Signal Processing (ICASSP). IEEE, 2018, pp. 1-5.

[21] https://github.com/xuchenglin28/speaker_ extraction/tree/master/simulation

[22] https://github.com/funcwj/conv-tasnet

[23] E. Vincent, R. Gribonval, and C. Févotte, "Performance measurement in blind audio source separation," IEEE transactions on audio, speech, and language processing, vol. 14, no. 4, pp. 1462-1469, 2006.

[24] J. Han, W. Rao, Y. Wang, and Y. Long, "Improving channel decorrelation for multi-channel target speech extraction," in Proc. Interspeech, 2021, pp. 1847-1851.

[25] R. Gu, J. Wu, S.-X. Zhang, L. Chen, Y. Xu, M. Yu, D. Su, Y. Zou, and D. Yu, "End-to-end multi-channel speech separation," arXiv preprint arXiv:1905.06286, 2019. 